\title{
Time-Varying Spatial Memory Model and Its Impact on Virus Spreading
}

\author{
Mingyue Xu $\mathbb{D},{ }^{1}$ Dingding Han $\mathbb{D},{ }^{1}$ Kaidi Zhao $\mathbb{D},{ }^{1}$ and Qingqing Yao ${ }^{2}$ \\ ${ }^{1}$ School of Information Science and Technology, Fudan University, Shanghai, China \\ ${ }^{2}$ School of Communication and Electronic Engineering, East China Normal University, Shanghai, China \\ Correspondence should be addressed to Dingding Han; ddhan@fudan.edu.cn
}

Received 16 October 2020; Revised 24 February 2021; Accepted 13 September 2021; Published 4 October 2021

Academic Editor: Ping Li

Copyright (c) 2021 Mingyue Xu et al. This is an open access article distributed under the Creative Commons Attribution License, which permits unrestricted use, distribution, and reproduction in any medium, provided the original work is properly cited.

The models of time-varying network have a profound impact on the study of virus spreading on the networks. On the basis of an activity-driven memory evolution model, a time-varying spatial memory model (TSM) is proposed. In the TSM model, the cumulative number of connections between nodes is recorded, and the spatiality of nodes is considered at the same time. Therefore, the active nodes tend to connect the nodes with high intimacy and close proximity. Then, the TSM model is applied to epidemic spreading, and the epidemic spreading on different models is compared. To verify the universality of the TSM model, this model is also applied to rumor spreading, and it is proved that it can also play a good inhibiting effect. We find that, in the TSM network, the introduction of spatiality and memory can slow down the propagation speed and narrow the propagation scope of disease or rumor, and memory is more important. We then explore the impact of different prevention and control methods on pandemic spreading to provide reference for COVID-19 management control and find when the activity of node is restricted, the spreading will be controlled. As floating population has been acknowledged as a key parameter that affects the situation of COVID-19 after work resumption, the factor of population mobility is introduced to calculate the interregional population interaction rate, and the time-varying interregional epidemic model is established. Finally, our results of infectious disease parameters based on daily cases are in good agreement with the real data, and the effectiveness of different control measures is evaluated.

\section{Introduction}

The transmission period of COVID-19 coincided with the Spring Festival travel rush on the eve of the Chinese New Year, during which a large population movement occurred throughout the country, expanding the spread of the virus and accelerating its spread $[1,2]$. The Chinese government adopted series of control measures to cut off the transmission path of the virus. After February 4, the number of reported daily cases in China began to decrease, indicating that the measures were working. Because of the certain negative impact on the lives of residents and social economy, some regions and enterprises began to return to work after February 10 , and the population mobility rate rose. The flowing of population made it more difficult to control the epidemic, so it was of great significance to study the way and modes of transmission of COVID-19.
From previous studies, we can learn about some epidemiological and clinical characteristics of COVID-19 [3, 4]. Compared with SARS and H1N1, the transmission of COVID-19 is stronger. The infection source is mainly a patient infected by COVID-19. A number of studies have proved that asymptomatic infected persons may also be the source of infection and that person-to-person contact is the main cause of virus transmission. Through analysis of early cases of COVID-19, Li et al. found that only $8.6 \%$ of the cases developed after January 1 were related to the seafood wholesale markets in South China, and human-to-human transmission had occurred among close contacts since midDecember 2019 [5]. Previous research has confirmed that contact is the main way for the spread of the virus, but in order to prevent and control the transmission of COVID-19 more effectively, further modeling and research are needed for the transmission dynamics of the virus. 
Many scholars have studied the development and policies of COVID-19 for further prevention and control from the perspective of epidemic dynamics [6,7]. Jia et al. [8] used the location data of mobile phone to track the flow of people from Wuhan to other areas and analyzed the transmission process of the pandemic based on the data. Kraemer et al. [9] used mobility data from Wuhan and case data including travel history to analyze the influence of travel restrictions on the spread of COVID-19 in China and found that travel restrictions in the early stages of the pandemic could be effective in preventing the importation of the virus. Moreover, complex network is an important tool for simulating transmission dynamics of virus or information on networks and can provide theoretical basis and guidance for it. COVID-19 has been analyzed using complex networks by some scholars [10]. Basnarkov [11] studied the SusceptibleExposed-Asymptomatic-Infectious-Recovered (SEAIR) epidemic spreading model to capture two characteristics: delayed start and its appearance before onset of symptoms. Some scholars have used multilayer networks to analyze the COVID-19 epidemic from the individual characteristics of different countries, such as social activities and economic characteristics $[12,13]$. Wang et al. [14] proposed a disease model based on two-layered multiplex networks, demonstrating that the epidemic prevalence and the epidemic outbreaks can be suppressed by the diffusion of positive preventive information and be promoted by the diffusion of negative preventive information. In addition, the dynamics of multiple information transmission and epidemic coevolution under the influence of mass media are discussed in [15].

The spread of COVID-19 is mostly analyzed from the perspective of overall population flow for epidemic prevention and control measures, but the establishment and transmission mode of interpersonal social network of COVID-19 spreading are lacking. In this paper, from the construction of a disease transmission network, we try to study COVID-19 from the perspective of transmission dynamics on the time-varying network.

In most real networks, individual attributes, such as activity and location, all change over time and lead to changes in network topologies on the macroscopic view. Therefore, it is more appropriate to use time-varying networks to simulate the structure of real networks [16]. Timevarying networks are widely used [17-19]. According to characteristics of social networks, $\mathrm{N}$ perra et al. verified degree distribution and weight distribution of nodes in the aggregated static network met the power-law distribution. The ratio of the number of activities of node $i$ to the number of activities of all nodes in a period of time was defined as the activity of node $i$, and it was found that the distribution of activity also met the power-law distribution. Therefore, $\mathrm{N}$ perra et al. proposed the activity-driven model to describe time-varying networks [20]. Many scholars have proposed improvements and optimizations to the time-varying network model, to make it more in line with the real network and dynamic characteristics, and mainly applied it to the epidemic transmission and the information transmission in social networks [21]. Some scholars set properties for nodes, such as attractiveness and willingness [22-29] or introduced external factor to the process of propagation [30-32].

We first try to build a network model closer to the real network. In the real network, the process of node connecting is not completely random, but the result of the non-Markov process with memory. Especially in social networks, social reinforcement derived from memory is the main feature of social contagions. It is of great practical significance to add memory mechanism [33-40] in time-varying networks. Moreover, it is known that, in our daily life, we tend to have more contact with the people around us or those close to us, while there is little work to consider spatial factors on the basis of the activity model.

Considering these characteristics, spatiality, activity, and memory of node are introduced into the construction of network and a time-varying spatial memory (TSM) model is proposed, which can better reflect real network characteristics. The TSM model is applied to the epidemic spreading to provide a reference for the spread, management, and control of the COVID-19 epidemic. Considering that infectious disease is the virus in biology, while rumor is the virus in sociology, this model is also applied to rumor spreading to prove the universality of the TSM model. Moreover, a time-varying interregional epidemic model under the influence of floating population is proposed to explore the impact of interregional population after returning to cities, and the influence and effect of different epidemic control measures are compared.

The remaining sections in this paper are organized on the basis of the following organization. In Section 2, a timevarying spatial memory model considering spatiality and memory of nodes is proposed based on the RP model. In Section 3, SEIR spreading and its simulation of the propagation of COVID-19 under the influence of floating population are studied. The TSM model is applied to the spread of the epidemic and also rumor, and the effect of control measures is analyzed in Section 4. In Section 5, an empirical study on the impact of returning to work in the epidemic situation is conducted. Last, conclusion is explained.

\section{Time-Varying Spatial Memory Network Model Based on the RP Model}

A model based on activity driven is proposed by $\mathrm{N}$ Perra et al., introducing the memory of nodes [40]. In this model, each node records all nodes that have been connected with it before time $t$ to form set $\mathrm{R}$. When active nodes establish edges at time $t$, the probability of selecting nodes in set $R$ increases with the increase of the number of nodes in it. However, for nodes belonging to set $R$, the probability of being selected is the same, which is also the limitation of the memory model. In the actual network, if the cumulative number of edges between pairs of nodes is taken as the edge weight, the weight will reflect the intimacy between nodes and have a great influence on the preference probability of connecting edges. For example, in social networks, the more the connections between individuals, the closer the relationship. Although some nodes have been connected, they 
may be only accidental. Obviously, we are more likely to spread information to friends and acquaintances and we cannot simply assume that the two categories are the same as in the RP model.

Meanwhile, both the spatiality and memory of nodes in time-varying networks will affect network structure. In social networks, for example, when we want to connect with others, we tend to choose the ones closer to us. Although the online social network is currently booming, if the distance between nodes is similar, the two are often in the same community. Distance is still one of the driving factors for node connection. On the other hand, we prefer to share with our close friends and partners when we get the news, rather than randomly choose one of the people we know.

Therefore, we consider both spatiality and memory of nodes in the model of time-varying networks. Nodes do not only record whether they have established a connection with other nodes but also record the total number of interactions with other nodes from the first time step and are more inclined to connect to nodes that are closer to them. These preferences are reflected in the preference probability of the destination node selected. The network model, named as the time-varying spatial memory (TSM) model, is constructed as follows:

(1) An activity $a_{i}$ is assigned to each node at the beginning of constructing the network, which is subject to the power-law distribution.

(2) In each discrete time step $\Delta t$, the initial state of network $G_{t}$ is composed of $\mathrm{N}$ disconnected nodes.

(3) Each node becomes active with probability $a_{i} \Delta t$. The active node creates $m$ edges and connects to other nodes. Inactive nodes can only receive edges. The probability of node $i$ choosing node $j$ to establish edges is as follows:

$$
P_{i j}= \begin{cases}\frac{n}{n+C} \cdot\left(\frac{\left(W_{i j}^{\alpha} / d_{i j}^{2}\right)}{\sum_{j \in R}\left(W_{i j}^{\alpha} / d_{i j}^{2}\right)}\right), & j \in R, \\ \frac{C}{n+C} \cdot\left(\frac{\left(1 / d_{i j}^{2}\right)}{\sum_{j \notin R, j \neq i}\left(1 / d_{i j}^{2}\right)}\right), & j \notin R,\end{cases}
$$

where $R$ is the set of nodes that have been connected to node $i . n$ is the number of nodes in set $R$. $C$ is the bias constant, and $C=1$ is set here. $W_{i j}$ is the cumulative number of edges between node pairs, $d_{i j}$ is the Manhattan distance between node $i$ and node $j$, and $\alpha$ is the proportion parameter. The larger the value of $\alpha$ is, the greater the influence of node memory on preference probability is.

As the number of connected nodes increases, the probability of connecting new nodes decreases. In [40], the author used $(C /(n+C))$ to define the probability of connecting a new node and showed that there would be a probability of $(n /(n+C))$ to connect an old node. In addition to the difference between the old and the new nodes, the probability of connecting the given two nodes increases as their intimacy increases, and whether it is a new node or an old node, the probability of creating an edge between two nodes decreases as the distance between them increases. So, after choosing the new node or the old node by probabilities $(C /(n+C))$ and $(n /(n+C))$, we set the probability of choosing a particular node as proportional to intimacy and inversely proportional to distance. Also, we use a parameter $\alpha$ to adjust the ratio between intimacy and distance.

According to the formula for the probability of connecting edges,

(1) The active node $i$ first decides whether to select the destination node in set $R$ according to the probability $P_{\text {oldneighbor }}(n)=(n /(n+C))$.

(2) If so, the destination node is selected according to the intimacy and distance between each node in set $R$ and node $i$. Otherwise, the closer node is randomly selected as the destination in the remaining new nodes.

(4) At the next time step $t+\Delta t$, all edges in the network $G_{t}$ are deleted and the second step is repeated to get the network $G_{t+\Delta t}$ at the next time step.

We analyze the degree distribution of the network generated by the TSM model and set $N=1000, L=32$, $m=3, a<0.8$, and $\alpha=0.5$. A network of 2000 time steps is generated, and every 400 time steps, the degree distribution of the current time step and the degree distribution of the integrated network superimposed at all times (including and excluding repeated edges) are recorded, as shown in Figure 1. Figure 1(a) shows the degree distribution of a single time step. As can be seen from the figure, the degree distribution of each time step is similar, and nodes with a degree value of 0 account for about $50 \%$ of the total nodes. Figure 1(c) shows the degree distribution of the integrated network. As time goes by, the number of edges in the network increases, and there are fewer nodes with small or large degree value, while there are more nodes with intermediate degree value. As can be seen from the figure, because the node activity in the network is the power-law distribution, it is difficult to be active for some nodes, and their cumulative degree values change slightly over time, while the degree values of nodes with higher activity increase significantly over time. Also, because the node considers the memory attribute when selecting the destination node, the difference between different time steps becomes smaller as time goes by. Figure 1(e) shows the degree distribution including the number of repeated edges, as there are repeated edges between a pair of nodes in the integrated network. In addition, we record the distribution of degree when the activity of nodes is set as 0.1 , as shown in Figures 1(b), $1(\mathrm{~d})$, and $1(\mathrm{f})$. The node distribution of a single time step in Figure 1(b) is similar to that in Figure 1(a), while in Figures $1(\mathrm{~d})$ and $1(\mathrm{f})$, due to the same node activity, the degree value increases evenly. 

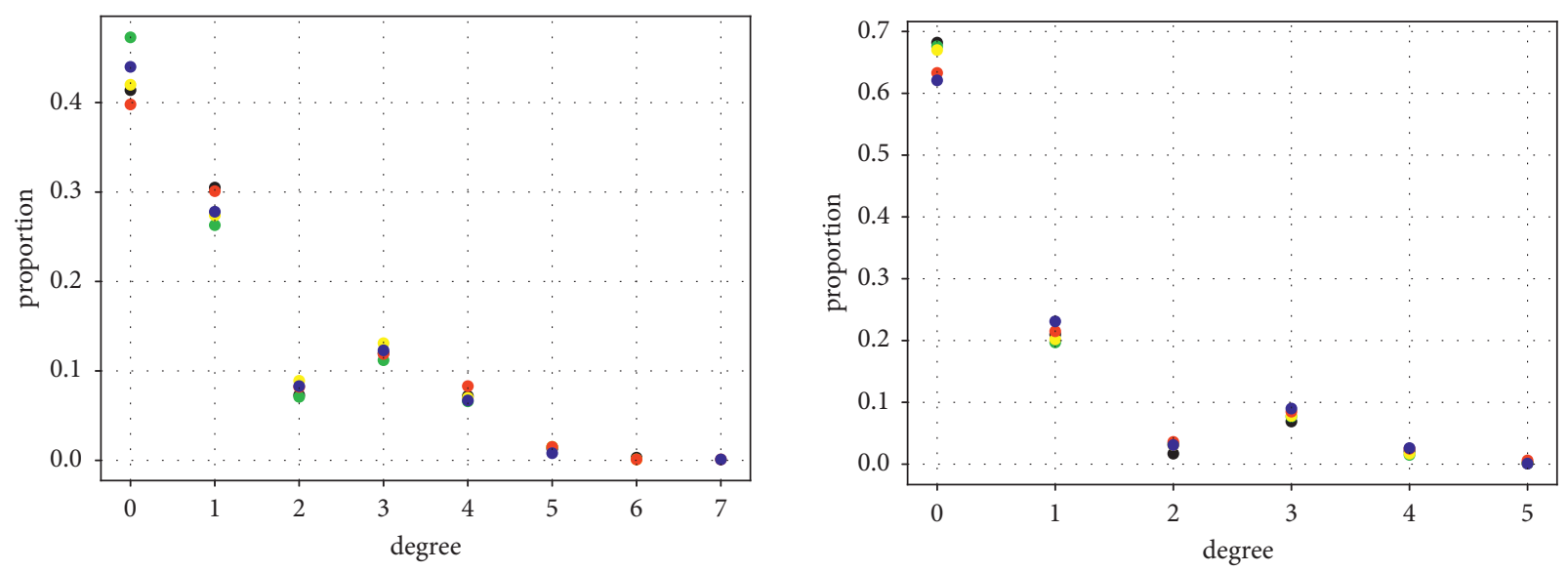

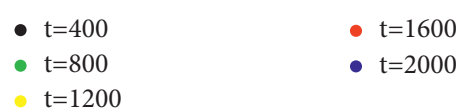

(a)
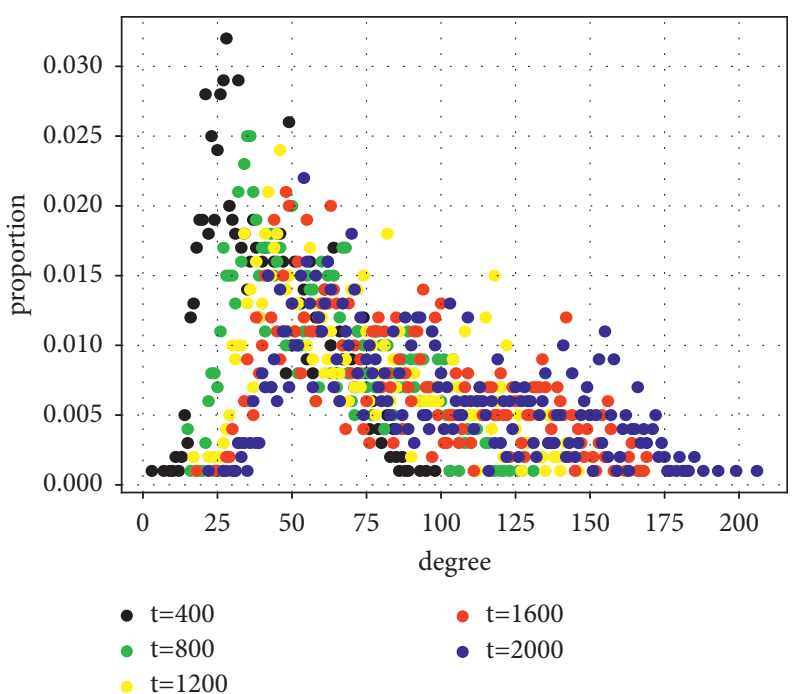

(c)

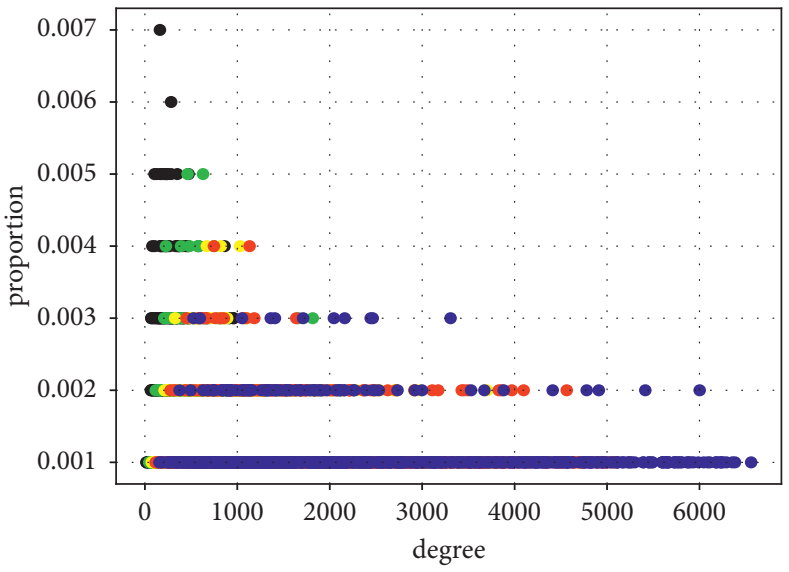

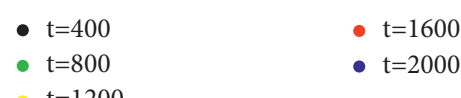

(b)

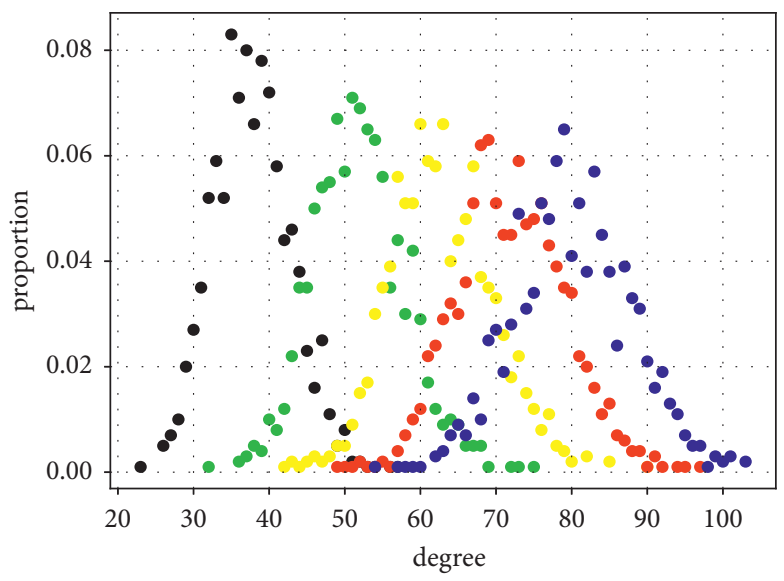

(d)

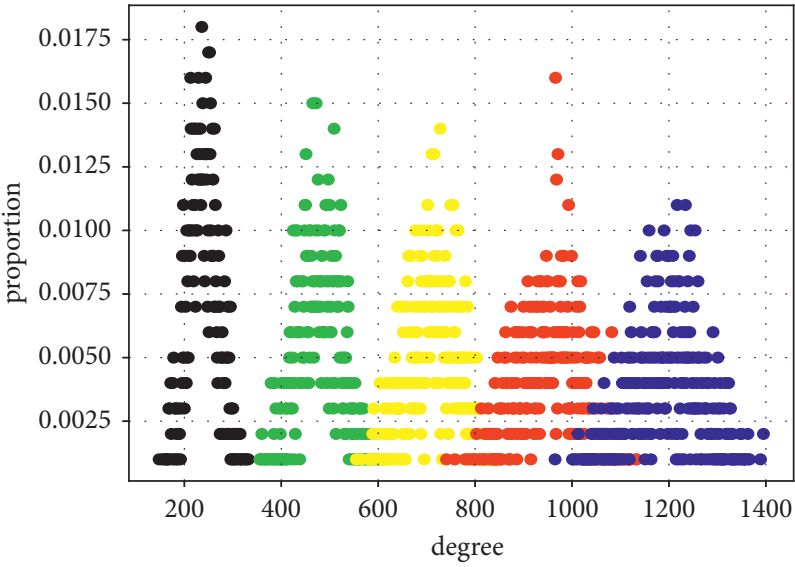

$$
\text { - } \begin{aligned}
& t=400 \\
& t=800 \\
& t=1200
\end{aligned}
$$

- $\mathrm{t}=1600$

- $t=2000$

(f)

Figure 1: Degree distribution of the network. (a) The degree distribution of a single time step. (c) The degree distribution of the integrated network excluding repeated edges. (e) The degree distribution of the integrated network including repeated edges. $N=1000, L=32, m=3$, $a<0.8$, and $\alpha=0.5$. (b) The degree distribution of a single time step when $a=0.1$. (d) The degree distribution of the integrated network excluding repeated edges when $a=0.1$. (f) The degree distribution of the integrated network including repeated edges when $a=0.1$. 
Because the TSM model comprehensively considers three driving attributes of nodes that affect the network topology and the proportion of driving factors that can be adjusted by parameter, it can be used as a general model framework for studying a complex system and its dynamic behaviors. The dynamics on the model also can apply to the spread of an epidemic and rumor.

\section{SEIR Spreading}

The epidemic model SEIR divides population into four categories: susceptible (S), exposed (E), infectious (I), and recover (R), and the four groups of individuals will transform or die at a certain rate. With the development of the epidemic and the improved control of COVID-19 after February 1, a variant of the SEIR model containing probable and isolated individuals [41] is adopted in this paper. $S_{k}, E_{k}, I_{k}, P_{k}, J_{k}, R_{k}$, respectively, label and quantify susceptible, exposed, infected, probable, isolated, and recovered individuals at time $t$. Then, the total population in the area $k$ at time $t$ is

$$
P_{\text {total }}=S_{k}+E_{k}+I_{k}+P_{k}+J_{k}+R_{k} \text {. }
$$

Probable individuals $P_{k}$ are quarantined individuals with confirmed epidemiological contact with a source of COVID19. It is assumed that all quarantined individuals include susceptible individuals and exposed individuals. When the exposed individuals develop symptoms, they become isolated individuals $J_{k}$, but their contact is reduced due to quarantine. So, even if they are exposed, they are not infectious, and after quarantine observation, asymptomatic groups will become susceptible groups and exposed groups will become isolated. Isolated population $J_{k}$ : infected persons become the isolated population through isolation or hospitalization, losing the ability to infect the susceptible population, which will be diminished due to recovery and disease-induced death.

We also consider the influence of population mobility. We suppose there are several geographical regions in addition to the current region, each region can be regarded as a community, and people from the same community are homogeneous; that is, they have the same biological and epidemiological parameters. According to the control measures for COVID-19, the time is divided into the period of strict control from February 1 to February 10 and the period of returning to work after February 10. Suppose that population flow between different regions is 0 from February 1 to February 10 because of strict controlling. After February 10 , due to the impact of work resumption, it is assumed that except the probable and the isolated who cannot leave the area, the remaining individuals can enter and leave the areas at a certain rate, but the flow rate of infected individuals is affected by epidemic control. Supposing that, after returning to work on February 10, the population inflow rate in the area $k$ is $\varphi_{k}$, the population outflow rate is $\rho_{k}$ and the limit coefficient of epidemic control on infected people is $c . c=0$ indicates that infected individuals cannot leave their areas, and $c=1$ indicates that infected individuals can move at the same rate as the susceptible. $S_{l}, E_{l}, I_{l}, R_{l}$ represent the total number of the susceptible, exposed, infected, and recovered individuals in other areas except region $k$, respectively.

The spread coefficients of exposed and infected persons are, respectively, $\beta_{E}$ and $\beta_{I}$, which represent the transmission probability of COVID-19. The infection rate of susceptible people in region $k$ is $\left(\left(\beta_{E} E_{k}+\beta_{I} E_{k}\right) S_{k} / P_{\text {total }}\right)$.

Since it is not possible to accurately distinguish COVID19 patients and ordinary influenza patients, ordinary influenza patients turn into probable individuals by probability $\theta_{S}$ and recover to susceptible individuals by probability $\theta_{P}$ after a period of isolation. $\gamma_{E}$ indicates the probability that an exposed individual will turn into a probable individual, and then, $\varepsilon_{E}$ means the exposed individuals will turn to infected with probability $\varepsilon_{E}$. $\xi_{I}$ refers to the self-healing rate of the infected individual, $\gamma_{I}$ represents the isolation rate of the infected individual in hospital, $\sigma$ represents the mortality rate of infected individuals, $\varepsilon_{P}$ represents the probability of the probable individuals becoming infected and being isolated, and $\xi_{J}$ indicates the cure rate of the hospitalized isolated population. We can get the equations of different groups of people in region $k$ :

$$
\begin{aligned}
\frac{\mathrm{d} S_{k}}{\mathrm{~d} t} & =\varphi_{k} S_{l}-\rho_{k} S_{k}-\frac{\left(\beta_{E} E_{k}+\beta_{I} I_{k}\right) S_{k}}{P_{\text {total }}}+\theta_{P} P_{k}-\theta_{S} S_{k}, \\
\frac{\mathrm{d} E_{k}}{\mathrm{~d} t} & =\varphi_{k} E_{l}-\rho_{k} E_{k}+\frac{\left(\beta_{E} E_{k}+\beta_{I} I_{k}\right) S_{k}}{P_{\text {total }}}-\varepsilon_{E} E_{k}-\gamma_{E} E_{k}, \\
\frac{\mathrm{d} I_{k}}{\mathrm{~d} t} & =c \varphi_{k} I_{l}-c \rho_{k} I_{k}+\varepsilon_{E} E_{k}-\gamma_{I} I_{k}-\xi_{I} I_{k}-\sigma I_{k}, \\
\frac{\mathrm{d} P_{k}}{\mathrm{~d} t} & =\gamma_{E} E_{k}-\theta_{P} P_{k}+\theta_{S} S_{k}-\varepsilon_{P} P_{k}, \\
\frac{\mathrm{d} J_{k}}{\mathrm{~d} t} & =\varepsilon_{P} P_{k}+\gamma_{I} I_{k}-\xi_{J} J_{k}-\sigma J_{k}, \\
\frac{\mathrm{d} R_{k}}{\mathrm{~d} t} & =\varphi_{k} R_{l}-\rho_{k} R_{k}+\xi_{I} I_{k}+\xi_{J} J_{k} .
\end{aligned}
$$

\section{The Impact of Time-Varying Network Models on Transmission Dynamics}

In real life, the network of contact between people changes over time. In order to explore the spread of COVID-19 on networks, the epidemic model in Section 3 is applied to the TSM model, and the influence of different time-varying network models is analyzed to prove the inhibition of the TSM model on propagation. Prevention and control strategies of epidemic on the spread of the epidemic are also analyzed.

When the epidemic model is applied to time-varying network models, the contact network between susceptible $S(t)$, exposed $E(t)$, infected $I(t)$, and recovered $R(t)$ is simulated. As we assume that the other two probable groups $P(t)$ and isolated groups $J(t)$ have little influence in the contact network, they are not discussed here. 
In a time-varying network, when a susceptible $(S)$ individual and an infected $(I)$ one meet, the susceptible may become exposed $(E)$ with probability $\beta_{I}$. If an exposed individual contacts with a susceptible one, the susceptible individual will become an exposed one with probability $\beta_{E}$; each time, exposed individuals turn infectious with probability $\varepsilon_{E}$ and the infectious individuals become recovered individuals with probability $\xi_{I}$. Figure 2 shows the propagation illustration of the model of epidemic spreading. In the figure, 25 nodes are taken as examples. Inactive nodes are represented by circles and active nodes are represented by stars.

The number of network nodes $N=10^{4}, L=100$, which means we distribute the nodes in a $100 \times 100$ grid in the simulation, the active nodes create $m$ edges each time where $m=3$, the minimum value of activity $a$ is set as 0.1 , the maximum value as 1.0, and the distribution of activity follows the power-law distribution with a power exponent of 0.78. Without loss of generality, we set $\beta_{I}=0.80, \beta_{E}=0.08, \varepsilon_{E}=0.10, \xi_{I}=0.10$, and $\alpha=0.50$ to get a clear view of the epidemic spreading.

The spread of the epidemic on the TSM model is simulated, as shown in Figure 3. There is a peak in the proportion of exposed and infected individuals. After 100 time steps, the proportion of the susceptible individuals decreases from 1 to 0 and the proportion of the recovered individuals increases from 0 to 1 .

To compare the difference of epidemic spreading between the TSM model and other time-varying network models and prove the inhibition of the TSM model on virus propagation, the process of epidemic spreading is also carried out on the ML model, IRP model considering only memory, and AD model considering only distance, respectively.

The probability of node $i$ selecting node $j$ of the IRP model is as follows:

$$
P_{i j}= \begin{cases}\frac{n}{n+C} \cdot \frac{W_{i j}}{\sum_{j \in R} W_{i j}}, & j \in R, \\ \frac{C}{n+C} \cdot \frac{1}{b}, & j \notin R,\end{cases}
$$

where $b$ is the number of nodes that node $i$ has not connected.

The probability of node $i$ selecting node $j$ of the AD model is as follows:

$$
P_{i j}=\frac{1}{d_{i j}^{2}} .
$$

The proportion of exposed people $E(t)$ in networks is shown in Figure 4. The AD model only considers the distance between nodes and only reduces the spread of the disease to a little extent. The IRP model considers the influence of memory and records the number of connections as the intimacy between nodes, which delays the spread of the epidemic and reduces the range of spreading. The TSM model reflects the contact feature more accurately because people tend to move around or get in touch with their close friends in general. Among the time-varying networks generated by the three models, the TSM network has the slowest spread of the epidemic and effectively suppresses it. In Figure 4, compared with the ML network reaching $57.14 \%$ of the maximum proportion of exposed persons, the TSM network reaches the maximum spread of $44.03 \%$. The proportion of exposed persons is reduced by about $13 \%$. This is because when a node chooses the destination node, it first divides the nodes into two categories by memory, which improves the probability of choosing old neighbors. At this time, the intimacy and distance between nodes work together, which makes the preference of connect stronger and the randomness weaker, and the "strong connection" and "weak connection" in the network become more obvious.

After the outbreak of COVID-19, a series of management and control measures were taken in China. One of the most important measures was to reduce the frequency of residents going out, to avoid the movement of people between regions, and to reduce personal contacts. In order to explore the effect of reducing human contact, different active mechanisms of nodes are simulated. First, the activity of nodes in the network is reduced. The maximum value is 0.8 , and the distribution of activity still follows a power-law distribution with a power exponent of 0.78 , making it more difficult for nodes to be active and reducing the number of active nodes to a certain extent. Figure 5(a) shows the proportion of infected nodes in the network. When the node activity is reduced and it is no longer easy to contact people, the proportion of infected people in the network is significantly reduced, and the time of the outbreak is delayed, from the maximum value of $31.7 \%$ to $21.61 \%$. The epidemic has been effectively prevented and controlled, which reflects the inhibition of strong connection.

Then, another possible situation about node activity is considered. The minimum value of activity a is set to 0.1 , and the distribution of activity still follows a power-law distribution with a power exponent of 0.78 , while the number of edges generated by active nodes is set to $m=1,2,3,4,5,6$, respectively. The results are shown in Figure 5(b). When the number of edges created by active nodes is reduced, fewer people are contacted in each time step, which also slows down the spread of the epidemic and the scope becomes smaller. When $m$ is set to 1 , the range of spread is reduced to about $15 \%$, and the peak of the outbreak is greatly delayed.

Due to rapid advances in medical technology for COVID-19, the cure rate of COVID-19 patients within a certain period of time has been effectively improved. Therefore, we assume that the probability of an infected person a becoming recovered person increases to 0.3 at each time step, and the rate of infected people is compared with that of $\xi_{I}=0.1$. The results are shown in Figure 5(c). Although the outbreak of the epidemic is hardly delayed, the number of people recovering from the infected increases at every moment, which has greatly reduced the number of infected people. Therefore, improving the cure probability of infected persons is also crucial to control the epidemic.

In order to explore the influence of the ratio of spatiality and memory in the TSM model on epidemic transmission, we compare the ratio of susceptible individuals in epidemic spreading under different $\alpha$, and the results are shown in Figure 6. It can be seen that the greater the value of $\alpha$ (i.e., the 

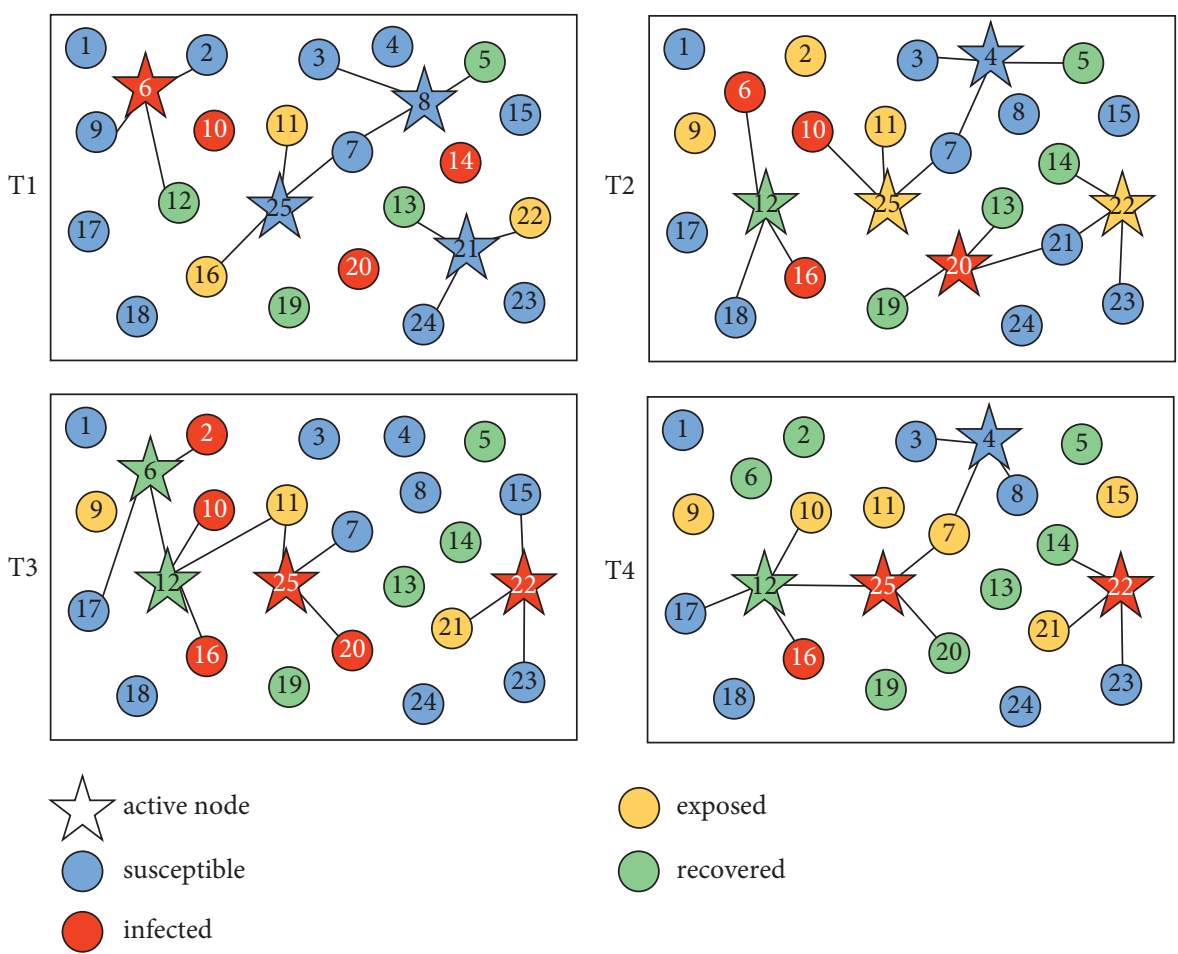

exposed

recovered

FIGURE 2: Schematic illustration of epidemic spreading on the TSM model. When a susceptible (S) individual and an infected (I) one meet, the susceptible may become exposed (E) with probability $\beta_{I}$. If an exposed individual contacts with a susceptible one, the susceptible individual will become an exposed one with probability $\beta_{E}$; each time, exposed individuals turn infectious with probability $\varepsilon_{E}$ and the infectious individuals become recovered individuals with probability $\xi_{I}$. T1 means the first time step, T2 means the second time step, and so on. 25 nodes are taken as examples in the illustration. The distribution of activity follows power-law distribution, as some nodes are often active, while others are rarely active.

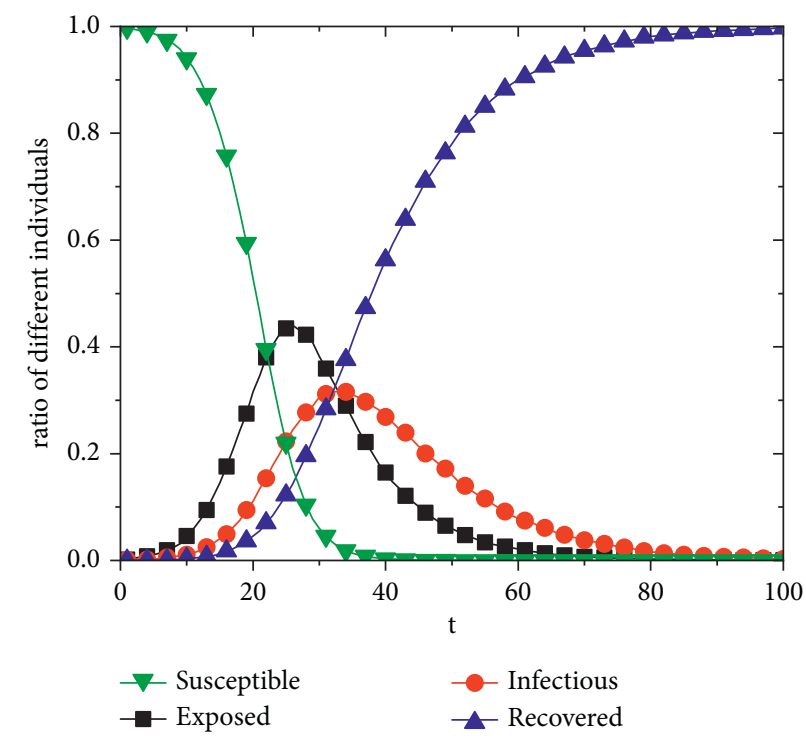

FIGURE 3: Behaviors of the epidemic spreading on the TSM model. There is a peak in the proportion of exposed and infected individuals. The proportion of susceptible individuals decreases from 1 to 0 , and the proportion of recovered individuals increases from 0 to $1 . N=10^{4}$, $L=100, m=3,0.1<a<1.0, \beta_{I}=0.80, \beta_{E}=0.08, \varepsilon_{E}=0.10, \xi_{I}=0.10$, and $\alpha=0.50$, and the distribution of activity follows a power-law distribution with a power exponent of 0.78 .

greater the dominance of memory over connection in the TSM model), the stronger the speed, and the scope of the epidemic spreading is inhibited. Specifically, when $\alpha=0$, the node selects the destination node in set $R$ only considering the distance. The range of epidemic spreading is close to $96 \%$. When $\alpha>0$, the intimacy affects the selection of the 


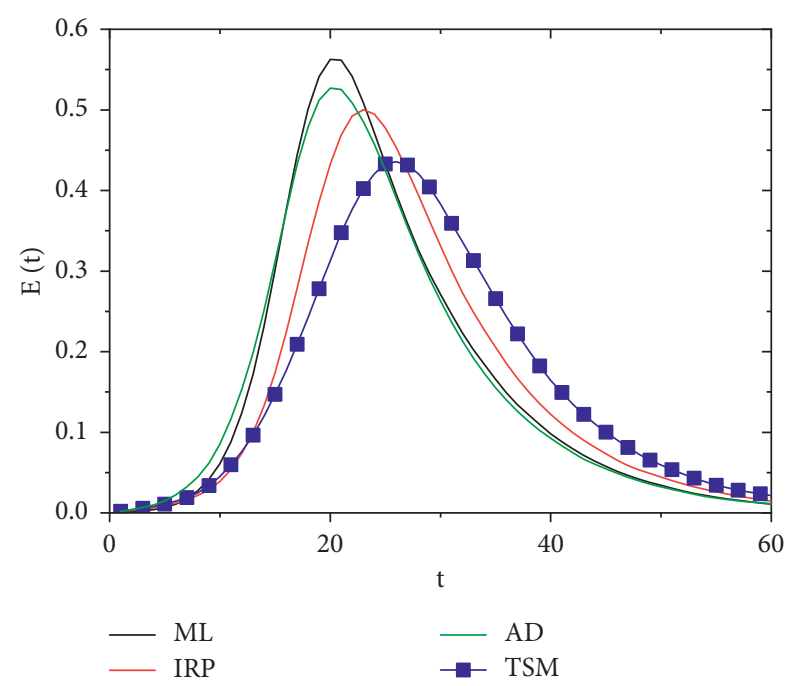

FIGURE 4: Comparison of proportions of exposed persons on timevarying networks generated by the four models. $N=10^{4}, L=100$, $m=3,0.1<a<1.0, \beta_{I}=0.80, \beta_{E}=0.08, \varepsilon_{E}=0.10, \xi_{I}=0.10$, and $\alpha=0.50$, and the distribution of activity follows a power-law distribution with a power exponent of 0.78 .

edge. With the increase of $\alpha$, the proportion of intimacy increases, and the inhibition effect on the speed and range of epidemic spreading is also gradually enhanced. For example, when $\alpha=4$, the spread range drops to about $66 \%$. Thus, it can be further verified that memory has a greater inhibitory effect on transmission compared with spatiality.

In order to verify whether the epidemic spreading on the time-varying network is consistent with the real epidemic spreading of COVID-19, the parameters are adjusted and the number of infected persons is compared with the real number of confirmed cases in Shanghai, Zhejiang, and Henan from February 1 to 29 [42]. It can be seen from Figure 7 that the propagation trend in time-varying networks and real networks is roughly the same. Because we adjust the parameters of different regions in the model, the transmission process is more in line with the real network. The number of infected people goes through a peak from low to high, and then, it declines with most of the people becoming immune. It is of great practical significance to study the propagation process on time-varying networks.

In order to further prove the universality of the TSM model, we also apply the model to the rumor propagation process in social networks. The spread of rumors is similar to the spread of viruses in some ways. Infectious disease is the virus in biology, while rumor is the virus in sociology.

There are three states of nodes in the network, namely, ignorant, spreader, and recover. In each time step, if the spreader interacts with the ignorant, the ignorant will become a spreader with the infection rate $\lambda$. In the event of an interaction between two spreaders or an interaction between the spreader and the recover, the person may be aware of the rumor or find that the information is known to the public, so the interest in continuing to transmit the information will decline and the spreader will become a recover at the recovery rate $\mu$. We set the parameters of the models as
$N=10^{5}, m=1$, and the distribution of activity follows a power-law distribution with a power exponent of $2.8, \lambda=1$, and recovery rate $\mu=0.6$. The rumor spreading process is observed on the network generated by the TSM model, and the results are compared with the ML model, AD model, and IRP model. Figure 8 shows the proportion of ignorant Ig $(t)$ with time step. The influence of each driving factor on propagation speed and range is analyzed.

The results of rumor propagation on networks are similar with that of the epidemic spreading. The results based on AD and ML are very similar. The rumor propagation speed in the AD model drops slightly, and there is basically no impact on the spreading ranges. The IRP model has an obvious constraint on the rumor spreading. In the TSM model, the propagation speed and range are further controlled. We adjust the parameters of the models to simulate a real mail network. The mail network is built from the mail exchange data of about 1000 staff in a European research institute in 803 days, and the data are from Stanford University's data website SNAP. The rumor propagation on various networks is shown in the subgraph of Figure 8. The network generated by the TSM model is the closest to the result of the actual network among several models.

Figure 9 shows the rumor propagation on the network generated by the TSM model when the proportion parameter $\alpha$ takes different values with the number of nodes $N=10^{4}$. Different with epidemic spreading, multiple connections between two nodes may cause the node to become recover more quickly. It may lead to the stronger inhibition effect of memory in the results.

\section{Impact of Work Resumption on COVID-19}

We collect the number of daily confirmed, existing confirmed, suspected, cured, and dead cases of COVID-19 in China as of February, 2020. The data come from reported cases in Chinese provinces online [42]. We estimate the parameters in the model by existing data and referring to existing work.

First, the parameters of the model from February 1 to 10 are estimated according to the work in $[5,43,44]$ and the data, and the following parameters are used for simulation: $\beta_{I}=0.1379, \beta_{E}=0.01379, \theta_{P}=0.04002, \theta_{S}=0.02213, \varepsilon_{E}=$ $0.02083, \quad \varepsilon_{P}=0.2, \gamma_{E}=0.1875, \gamma_{I}=0.3448, \xi_{I}=0.002, \xi_{I}$ $=0.03, \sigma=0.0021$. We assume the epidemic control is strict during the period from February 1 to February 10; then, $\varphi_{k}=0, \rho_{k}=0, c=0$. Numerical simulation of daily COVID19 patients in Shanghai is shown in Figure 10.

Although strong prevention and control measures have played an important role in curbing the outbreak of COVID19 , these measures have also had a huge negative impact on daily life and economic development. Many regions and enterprises start work resumption after February 10, which also means that the population flow begins to increase and may lead to a renewed COVID-19 outbreak. To solve this problem, a simulation is carried out by controlling the daily inflow and outflow of population. $\delta$ represents the ratio of daily inflow and outflow to the local population. Assuming 


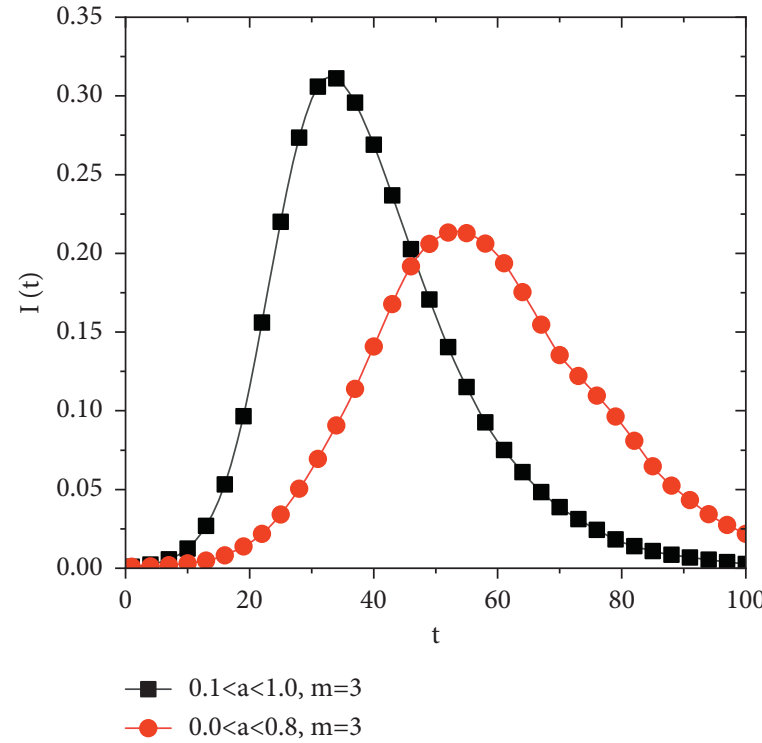

(a)

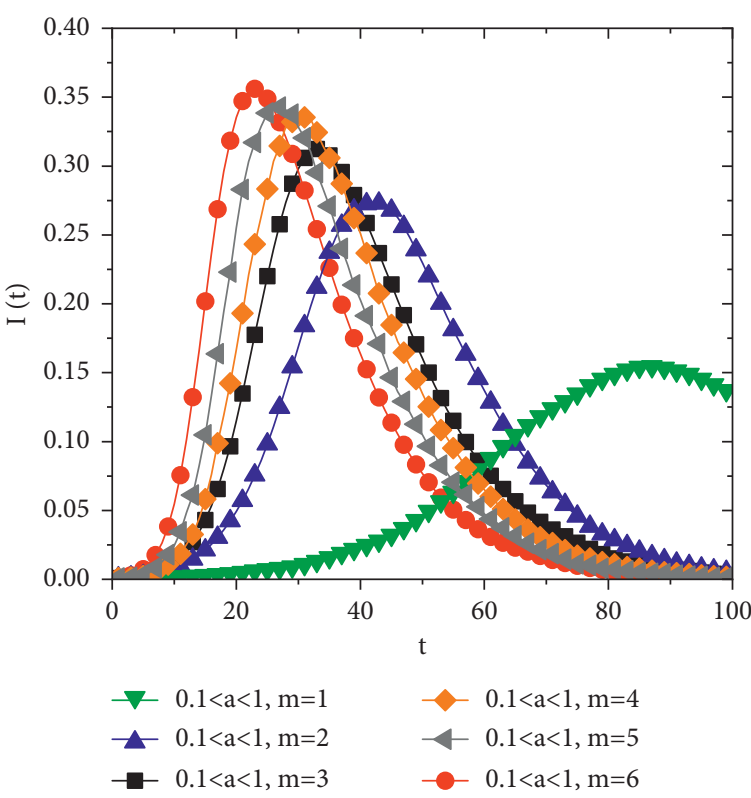

(b)

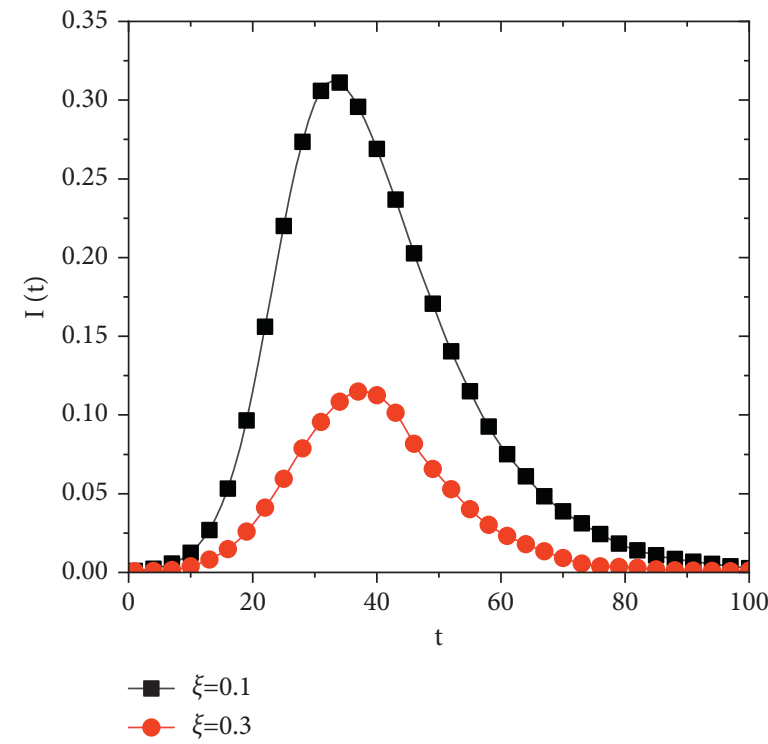

(c)

Figure 5: Comparison of infected persons under different management and control measures. (a) Proportion of infected persons before and after reducing node activity. The activity $a$ is set to $0.1<a<1.0$ and $0.0<a<0.8$, respectively, and the distribution of activity still follows a power-law distribution with a power exponent of 0.78 . (b) Proportion of infected persons with different number of edges created by active nodes, $m=1,2,3,4,5,6$, respectively. (c) Proportion of infected persons before and after improving the recovery probability, $\xi_{I}=0.1,0.3$, respectively.

that the distribution of various groups of people in various regions is similar, there are

$$
\delta=\frac{\varphi_{k} S_{l}-\rho_{k} S_{k}}{S_{k}}=\frac{\varphi_{k} E_{l}-\rho_{k} E_{k}}{E_{k}}=\frac{\varphi_{k} R_{l}-\rho_{k} R_{k}}{R_{k}} .
$$

The movement of infected people $\delta_{I}$ is affected by epidemic control:

$$
\delta_{I}=\frac{c \varphi_{k} I_{l}-c \rho_{k} I_{k}}{I_{k}}=c \delta
$$

where $S_{k}, E_{k}, I_{k}, P_{k}, J_{k}, R_{k}$, respectively, represent susceptible, exposed, infected, probable, isolated, and recovered individuals in the area $k, S_{l}, E_{l}, I_{l}, R_{l}$ represent the total number of susceptible, exposed, infected, and recovered individuals in other areas except region $k$, respectively. The population 


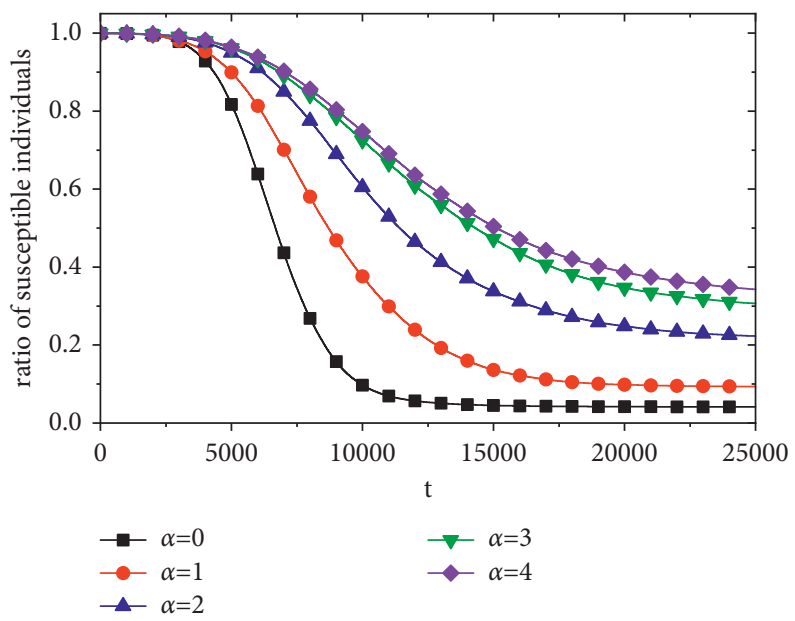

Figure 6: The impact of different $\alpha$ on epidemic spreading. $\alpha=0,1,2,3,4,5$, respectively. The greater the value of $\alpha$, the greater the proportion of memory. $N=10^{4}, L=100, m=1,0.0<a<0.8, \beta_{I}=0.8, \beta_{E}=0.08, \varepsilon_{E}=0.08, \xi_{I}=0.0004$, and the distribution of activity follows a power-law distribution with a power exponent of 2.8. The greater the value of $\alpha$, the stronger the speed, and the scope of the epidemic spreading is inhibited.

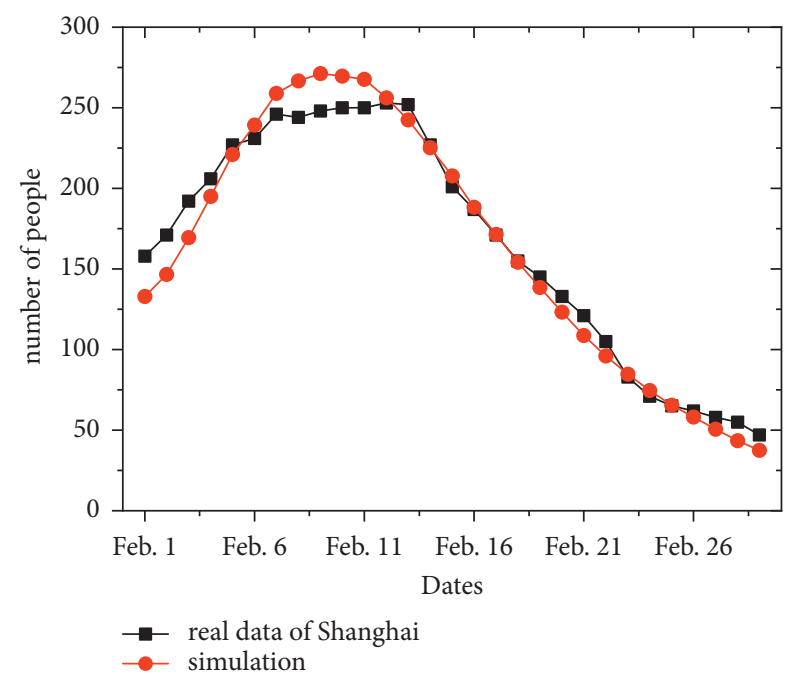

(a)

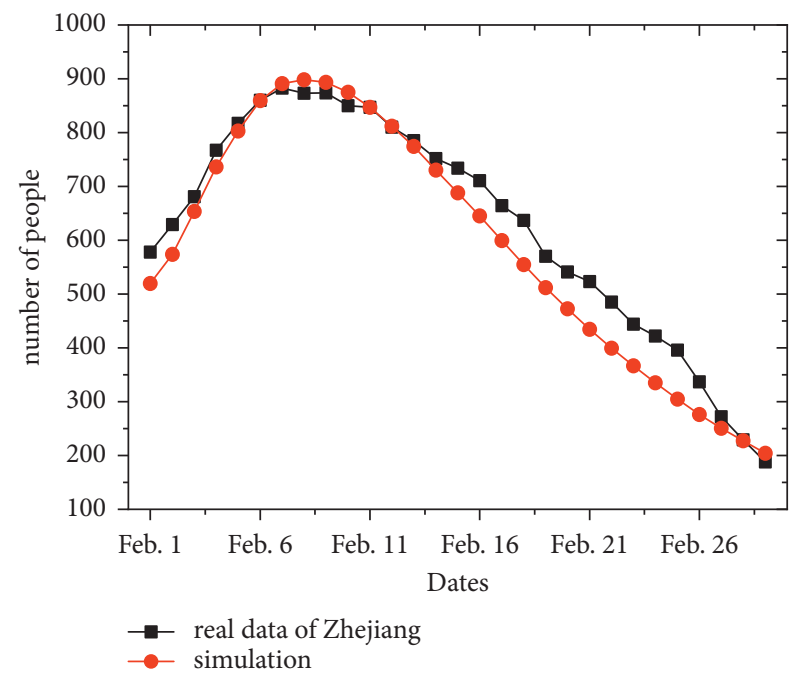

(b)

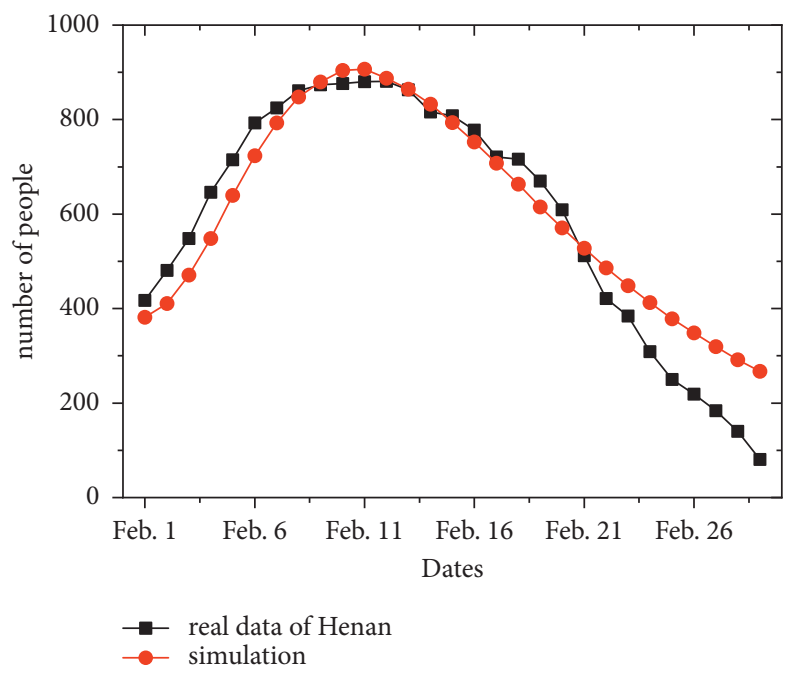

(c)

FIGURE 7: The simulated number of infected persons on the TSM model and the real number of confirmed cases in three regions in February, 2020. (a) Simulated and real data of Shanghai. (b) Simulated and real data of Zhejiang. (c) Simulated and real data of Henan. The propagation trend in time-varying networks and real networks is roughly the same. It is of great practical significance to study the propagation process on time-varying networks. 


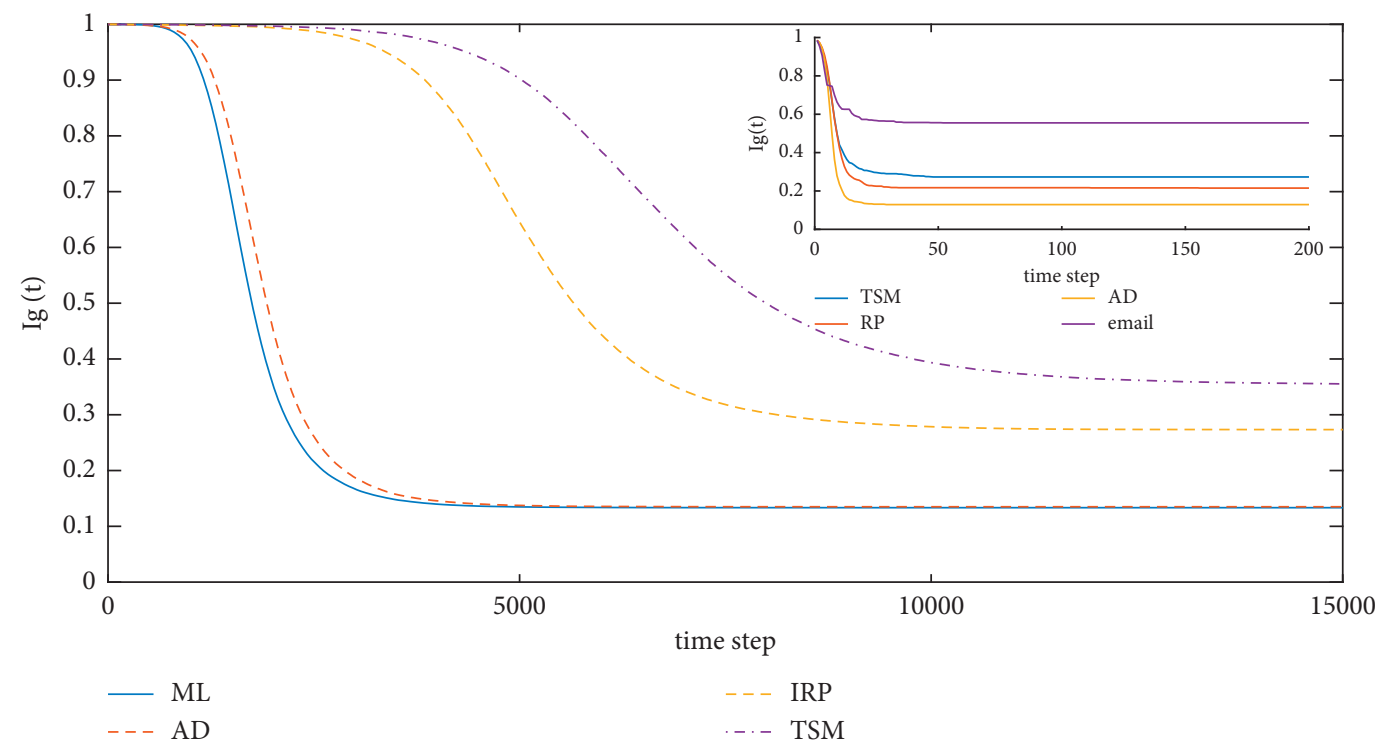

FIGURE 8: Ig $(t)$ over time during rumor propagation on the network of ML, AD, IRP, and TSM models. The rumor propagation speed in the $\mathrm{AD}$ model drops slightly, and there is basically no impact on the spreading ranges. The IRP model has an obvious constraint on rumor spreading. In the TSM model, the propagation speed and range are further controlled. Subgraph is the comparison of rumor propagation results between the simulated network and actual mail network. $N=10^{5}, m=1, \lambda=1$, and $\mu=0.6$, and the distribution of activity follows a power-law distribution with a power exponent of 2.8 .

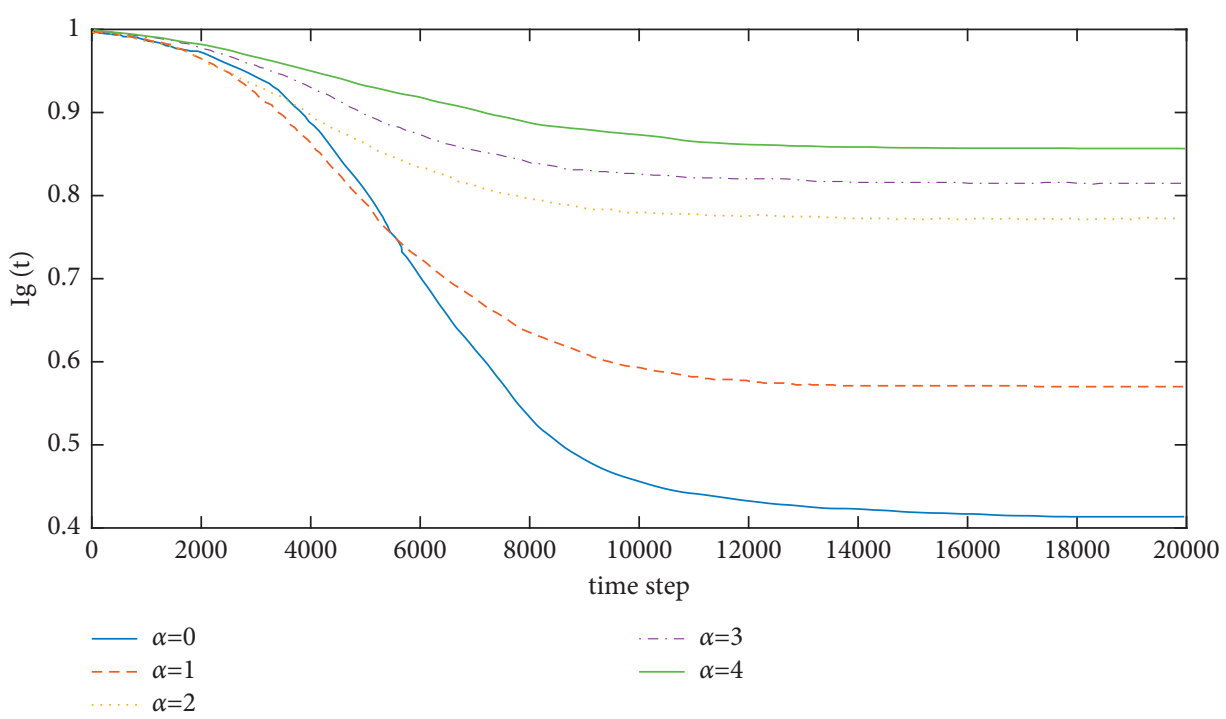

FIgURE 9: The influence of different $\alpha$ on the rumor propagation on the network. The inhibition effect of memory is more stronger. $\alpha=0,1,2,3,4,5$, respectively, $N=10^{5}, m=1, \lambda=1$, and $\mu=0.6$, and the distribution of activity follows a power-law distribution with a power exponent of 2.8 .

inflow rate in the area $k$ is $\varphi_{k}$, and the population outflow rate is $\rho_{k} . \delta<0$ indicates that the outflow of population is greater than the inflow of population, while $\delta>0$ indicates the opposite. Taking Shanghai as an example, the epidemic trend under different inflow and outflow rates is simulated, and the results are shown in Figure 11. It is found that, after the epidemic has been basically controlled, the impact of urban population decreasing on the epidemic situation is small. For cities with an influx of population, $\delta$ has little impact on the epidemic when it is relatively small $(\delta \leq 0.14)$. However, as $\delta$ is gradually increasing, the epidemic is getting serious. It will lead to the outbreak of the epidemic again in a short time when $\delta \geq 0.20$, so it is important to control the population flow during the return journey.

In order to study the influencing factors of work resumption, we analyze the comprehensive effects of the delay in returning and the inflow rate on the epidemic. It can be found from Figure 12(a) that returning to the city at a higher 


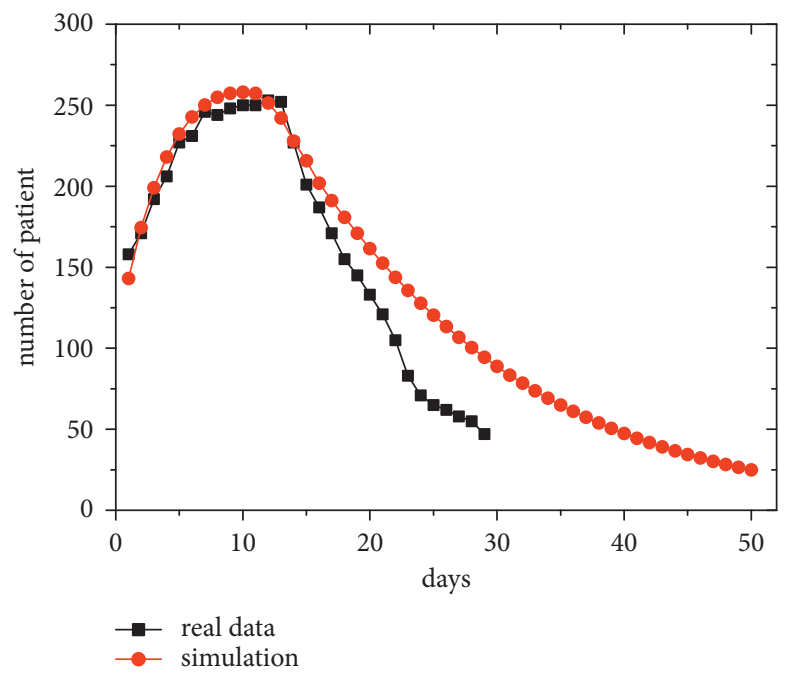

FIGURE 10: The simulated result of the time-varying interregional epidemic model and the real number of confirmed cases in Shanghai in February, 2020. The red curve indicates the actual number of COVID-19 patients per day. The black curve is simulated by the interregional model. In February, our simulation results fit well with the real results, and we also made predictions about the epidemic situation after February.

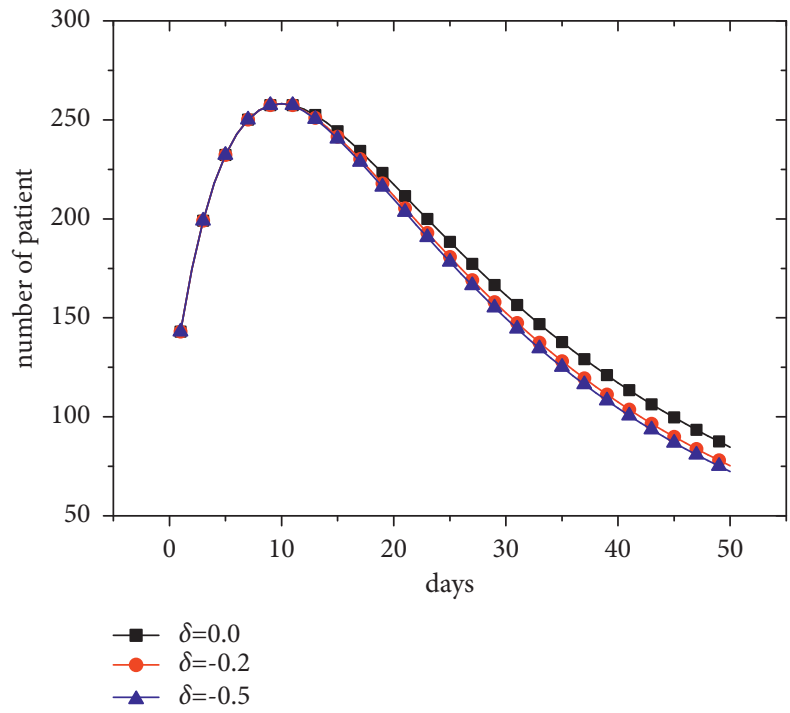

(a)

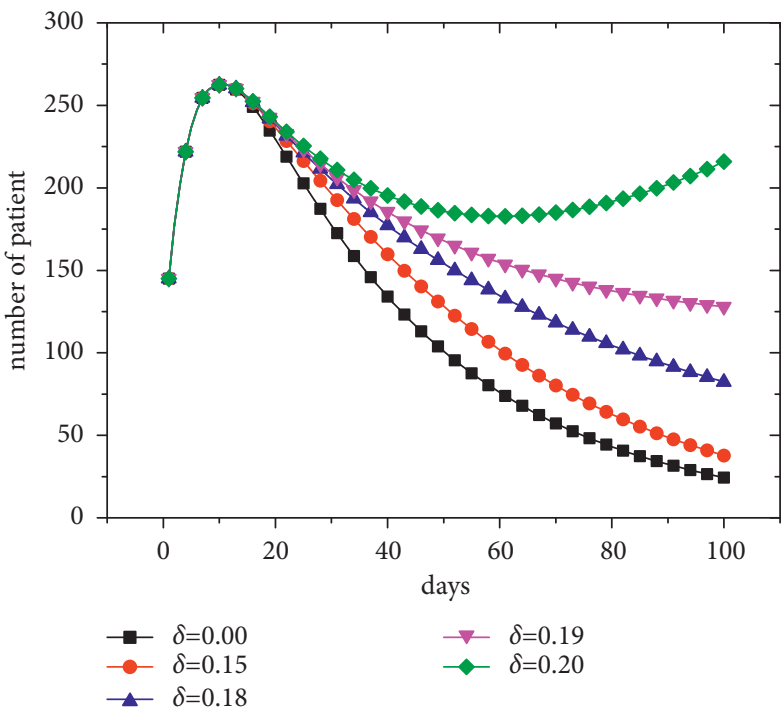

(b)

Figure 11: The impact of different rates on the epidemic. (a) The impact of different outflow rates on the epidemic when the outflow of population is greater. After the epidemic has been basically controlled, the impact of the urban population decreasing on the epidemic situation is small. (b) The impact of different inflow rates on the epidemic when the inflow of population is greater, and the $x$-axis is extended to 100 days to see the trend. $\delta$ has little impact on the epidemic when it is relatively small $(\delta \leq 0.14)$. While varying $\delta$ from 0.14 to 0.20 , the epidemic is getting serious. Also, it will lead to the outbreak of the epidemic again in a short time when $\delta \geq 0.20$.

rate of population inflow after 7 days can effectively reduce the epidemic. We further consider the impact of returning to the city in batches, and the result in Figure 12(a) indicates that returning to the city in batches can ensure the efficiency of resumption of work and avoid a great impact on the epidemic.

Due to the lack of reliable and efficient virus detection methods, human errors, and some unregulated population inflow, it is impossible to effectively and accurately detect and isolate all infected people returning to work. Figure 12(b) shows the epidemic trend under two different migration policies ( $c=0$ and $c=1$ ) with different $\delta$. It is easy to find from Figure 12(b) that even if all infected persons are effectively isolated, it will be of little help to control the epidemic under the increasing inflow of population. The results show that when there are enough exposed persons, 


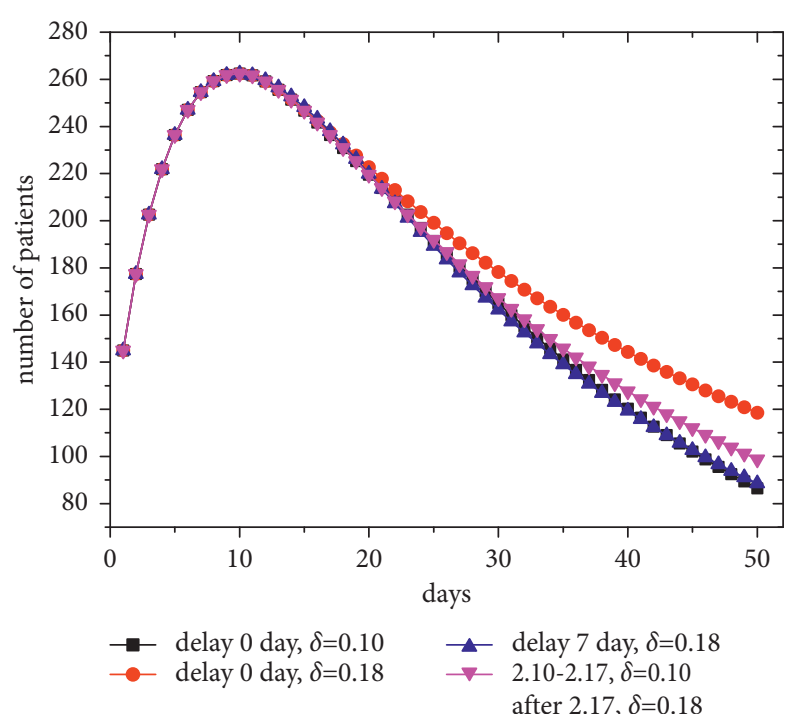

(a)

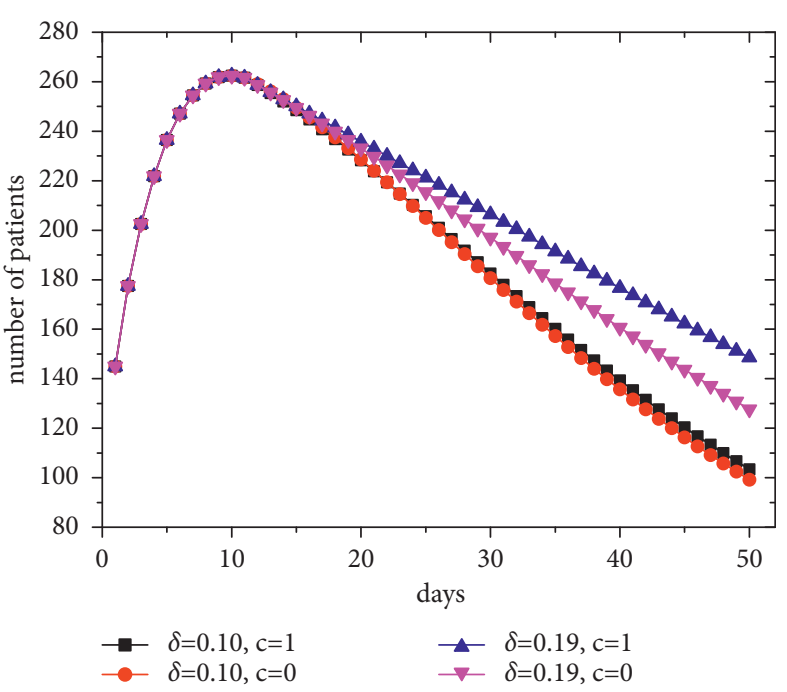

(b)

Figure 12: The trend of the epidemic under different conditions. (a) The relationship between postponing the resumption of work and increasing population inflow. Returning to the city at a higher rate of population inflow after 7 days can effectively reduce the epidemic, and returning to the city in batches can also ensure the efficiency of resumption of work and avoid a great impact on the epidemic. (b) The trend of the epidemic under different $\delta$ and $c$. When there are enough exposed persons, even if the infected people are completely isolated, the development of the epidemic cannot be effectively prevented.

even if the infected people are completely isolated, the development of the epidemic cannot be effectively prevented.

\section{Conclusions}

Considering that the spatiality and memory attribute of nodes are two important driving factors to promote the evolution of time-varying networks besides activity, we propose the TSM model by combining the distance and memory. If the cumulative interaction between two nodes is more or the two nodes are closer, the probability of establishing edges between them is greater. The proportion of the two driving factors in the model can be adjusted by parameters, so that the model can be adapted to a variety of practical time-varying networks.

In order to provide a reference for the spread and management of COVID-19, we apply the TSM model to pandemic spreading. The distance and intimacy between nodes simultaneously are driven by the network structure in the TSM model, so the epidemic spreading on the network is further reduced and slowed down in scope and speed. We also find that the memory is more important in inhibiting the spreading. Considering the requirements of epidemic prevention and management, we limit the active behaviors of nodes. The results show that when the activity of nodes decreases or the contacts created by active nodes per time step is reduced, the epidemic will be effectively controlled. In addition, increasing the recovery probability of infected people in each time step can greatly reduce the spread of the epidemic. We also verify the validity of the experiment on time-varying networks for epidemic spreading through real data, and the simulation result is the same as the trend of the real data. We then find that our model is also applicable to rumor propagation.

Finally, in order to understand the impact of interregional population flow caused by work resumption on the epidemic, the model based on the population mobility rate is proposed. We determine the relevant parameters of the epidemiological characteristics of COVID-19 through relevant literature and daily cases and prove that the model and parameters can fit the real data well. Then, the effectiveness of different control measures is evaluated, such as changing the flow rate, delaying the time to return to work, and strengthening the screening of inflows. Furthermore, we analyze the relationship between return time and virus transmission and find that delaying return time can reduce the spread of the epidemic and an appropriate $\delta$ increasing with the return time can have a less impact on the epidemic. In order to take into account the economic development at the same time, we suggest that we should let workers return to work in cities while controlling the epidemic, and the strategy of resuming work in batches can be implemented.

In this paper, we focus on the research of modeling the temporal interpersonal contact network of infectious disease and reflecting COVID-19 dynamics, while the threshold of the spreading or other characteristics are important from the overall perspective of epidemic spreading. The stability or critical characteristics of the proposed epidemic model deserves further investigation in the future.

\section{Data Availability}

The data used to support the findings of this study are available from the corresponding author upon request. 


\section{Conflicts of Interest}

The authors declare no conflicts of interest.

\section{Acknowledgments}

This work was supported by the National Natural Science Foundation of China (Grant nos. 11875133 and 11075057) and the National Key Research and Development Program of China (Grant no. 2018YFB2101302).

\section{References}

[1] World Health Organization, Coronavirus, WHO, Geneva, Switzerland, 2020, https://www.who.int/health-topics/ coronavirus $\backslash$ enleadertwodots.

[2] S. Zhao, Z. Zhuang, J. Ran et al., "The association between domestic train transportation and novel coronavirus (2019ncov) outbreak in China from 2019 to 2020: a data-driven correlational report," Travel Medicine and Infectious Disease, vol. 33, Article ID 101568, 2020.

[3] C. Huang, Y. Wang, X. Li et al., "Clinical features of patients infected with 2019 novel coronavirus in wuhan, China," The Lancet, vol. 395, no. 10223, pp. 497-506, 2020.

[4] J. F.-W. Chan, S. Yuan, K.-H. Kok et al., "A familial cluster of pneumonia associated with the 2019 novel coronavirus indicating person-to-person transmission: a study of a family cluster," The Lancet, vol. 395, no. 10223, pp. 514-523, 2020.

[5] Q. Li, X. Guan, P. Wu et al., "Early transmission dynamics in wuhan, China, of novel coronavirus-infected pneumonia," New England Journal of Medicine, vol. 382, no. 13, pp. 1199-1207, 2020.

[6] Y. Yin, J. Gao, B. F. Jones, and D. Wang, "Coevolution of policy and science during the pandemic," Science, vol. 371, no. 6525 , pp. 128-130, 2021.

[7] X. Hao, S. Cheng, D. Wu, T. Wu, X. Lin, and C. Wang, "Reconstruction of the full transmission dynamics of covid-19 in wuhan," Nature, vol. 584, no. 7821, pp. 420-424, 2020.

[8] J. S. Jia, X. Lu, Y. Yuan, G. Xu, J. Jia, and N. A. Christakis, "Population flow drives spatio-temporal distribution of covid19 in China," Nature, vol. 582, no. 7812, pp. 389-394, 2020.

[9] M. U. G. Kraemer, C.-H. Yang, B. Gutierrez et al., "The effect of human mobility and control measures on the covid-19 epidemic in China," Science, vol. 368, no. 6490, pp. 493-497, 2020.

[10] A. Arenas, W. Cota, J. Gómez-Gardeñes et al., "Modeling the spatiotemporal epidemic spreading of covid-19 and the impact of mobility and social distancing interventions," Physical Review X, vol. 10, no. 4, Article ID 41055, 2020.

[11] L. Basnarkov, Epidemic Spreading Model of Covid-19, 2020.

[12] L. F. S. Scabini, L. C. Ribas, M. B. Neiva, A. G. B. Junior, A. J. F. Farfán, and O. M. Bruno, "Social interaction layers in complex networks for the dynamical epidemic modeling of covid-19 in Brazil," Physica A, vol. 564, Article ID 125498, 2021.

[13] E. Montes-Orozco, R.-A. Mora-Gutiérrez, S.-G. De-LosCobos-Silva et al., "Identification of covid-19 spreaders using multiplex networks approach," IEEE Access, vol. 8, pp. 122874-122883, 2020.

[14] Z. Wang, C. Xia, Z. Chen, and G. Chen, "Epidemic propagation with positive and negative preventive information in multiplex networks," IEEE transactions on cybernetics, vol. 51, no. 3, pp. 1454-1462, 2020.
[15] Z. Wang and C. Xia, "Co-evolution spreading of multiple information and epidemics on two-layered networks under the influence of mass media," Nonlinear Dynamics, vol. 102, no. 4, pp. 3039-3052, 2020.

[16] P. Holme and J. Saramäki, “Temporal networks," Physics Reports, vol. 519, no. 3, pp. 97-125, 2012.

[17] L. E. C. Rocha, "Dynamics of air transport networks: a review from a complex systems perspective," Chinese Journal of Aeronautics, vol. 30, no. 2, pp. 469-478, 2017.

[18] S. Sarkar, S. Sikdar, S. Bhowmick, and A. Mukherjee, "Using core-periphery structure to predict high centrality nodes in time-varying networks," Data Mining and Knowledge Discovery, vol. 32, no. 5, pp. 1368-1396, 2018.

[19] Q. Chen, J. H. Qian, L. Zhu, and D. D. Han, "Optimal transport in time-varying small-world networks," Physical Review, vol. 93, no. 3, Article ID 32321, 2016.

[20] N. Perra, B. Gonçalves, R. Pastor-Satorras, and A. Vespignani, "Activity driven modeling of time varying networks," Scientific Reports, vol. 2, no. 1, p. 469, 2012.

[21] Y. Moreno, M. Nekovee, and A. F. Pacheco, "Dynamics of rumor spreading in complex networks," Physical review. E, Statistical, nonlinear, and soft matter physics, vol. 69, no. 6, Article ID 66130, 2004.

[22] L. Alessandretti, K. Sun, A. Baronchelli, and N. Perra, "Random walks on activity-driven networks with attractiveness," Physical Review, vol. 95, no. 5, Article ID 52318, 2017.

[23] A. Moinet, A. Barrat, and R. Pastor-Satorras, "Generalized voterlike model on activity-driven networks with attractiveness," Physical Review, vol. 98, no. 2, Article ID 22303, 2018.

[24] M. Starnini, A. Baronchelli, and R. Pastor-Satorras, "Modeling human dynamics of face-to-face interaction networks," Physical Review Letters, vol. 110, no. 16, Article ID 168701, 2013.

[25] T. Aoki, L. E. Rocha, and T. Gross, "Temporal and structural heterogeneities emerging in adaptive temporal networks," Physical Review, vol. 93, no. 4, Article ID 40301, 2016.

[26] J. Wang, J. Xu, Y. Liu, and W. Deng, "AST: activity-securitytrust driven modeling of time varying networks," Scientific Reports, vol. 6, no. 1, p. 21352, 2016.

[27] H. Dun, Y. Shuting, H. She, Q. Lingfei, and A. B. Chris, "Research on how the difference of personal propagation ability influences the epidemic spreading in activity-driven network," Physica A: Statistical Mechanics and Its Applications, vol. 514, pp. 311-318, 2019.

[28] X. An, L. Ding, and P. Hu, "Information propagation with individual attention-decay effect on activity-driven networks," Physica A: Statistical Mechanics and Its Applications, vol. 556, Article ID 124815, 2020.

[29] L. Han, Z. Lin, M. Tang, J. Zhou, Y. Zou, and S. Guan, “Impact of contact preference on social contagions on complex networks," Physical Review, vol. 101, no. 4, Article ID 42308, 2020.

[30] F. Baumann, P. Lorenz-Spreen, I. M. Sokolov, and M. Starnini, "Modeling echo chambers and polarization dynamics in social networks," Physical Review Letters, vol. 124, no. 4, Article ID 48301, 2020.

[31] Y. Cheng, C. Liu, and F. Ding, "Dynamic analysis of rumor spreading model for considering active network nodes and nonlinear spreading rate," Physica A: Statistical Mechanics and its Applications, vol. 506, pp. 24-35, 2018.

[32] H. Zhu, J. Ma, and S. Li, "Effects of online and offline interaction on rumor propagation in activity-driven networks," Physica A: Statistical Mechanics and its Applications, vol. 525, pp. 1124-1135, 2019. 
[33] H. Kim, M. Ha, and H. Jeong, "Impact of temporal connectivity patterns on epidemic process," The European Physical Journal B, vol. 92, no. 7, p. 161, 2019.

[34] M. Tizzani, S. Lenti, E. Ubaldi, A. Vezzani, C. Castellano, and R. Burioni, "Epidemic spreading and aging in temporal networks with memory," Physical Review E, vol. 98, no. 6, Article ID 62315, 2018.

[35] C. L. Vestergaard, M. Génois, and A. Barrat, "How memory generates heterogeneous dynamics in temporal networks," Physical Review E, Statistical, Nonlinear, and Soft Matter Physics, vol. 90, no. 4, Article ID 42805, 2014.

[36] Y. Zhou, Z. Yue, Q.-H. Liu, and W. Wang, "Social reinforcement inducing discontinuous spreading in complex networks," EPL (Europhysics Letters), vol. 128, no. 6, p. 68002, 2020.

[37] O. E. Williams, F. Lillo, and V. Latora, "Effects of memory on spreading processes in non-markovian temporal networks," New Journal of Physics, vol. 21, no. 4, Article ID 43028, 2019.

[38] H. Kim, M. Ha, and H. Jeong, "Scaling properties in timevarying networks with memory," The European Physical Journal B, vol. 88, no. 12, p. 315, 2015.

[39] H. Kim, M. Ha, and H. Jeong, "Dynamic topologies of activity-driven temporal networks with memory," Physical Review, vol. 97, no. 6, Article ID 62148, 2018.

[40] M. Karsai, N. Perra, and A. Vespignani, "Time varying networks and the weakness of strong ties," Scientific Reports, vol. 4, no. 1, p. 4001, 2014.

[41] A. B. Gumel, S. Ruan, T. Day et al., "Modelling strategies for controlling sars outbreaks," Proceedings of the Royal Society of London. Series B: Biological Sciences, vol. 271, no. 1554, pp. 2223-2232, 2004.

[42] E. Dong, H. Du, and L. Gardner, "An interactive web-based dashboard to track covid-19 in real time," The Lancet Infectious Diseases, vol. 20, no. 5, pp. 533-534, 2020.

[43] J. T. Wu, K. Leung, and G. M. Leung, "Nowcasting and forecasting the potential domestic and international spread of the 2019-ncov outbreak originating in wuhan, China: a modelling study," The Lancet, vol. 395, no. 10225, pp. 689-697, 2020.

[44] J. Wallinga and M. Lipsitch, "How generation intervals shape the relationship between growth rates and reproductive numbers," Proceedings of the Royal Society B: Biological Sciences, vol. 274, no. 1609, pp. 599-604, 2007. 